\title{
Rapid and Automated Extraction of the Fourth Ventricle from MR Images
}

\author{
Yan Xia, Aamer Aziz, QingMao Hu, and Wieslaw L. Nowinski \\ Biomedical Imaging Lab, Institute for Infocomm Research \\ 21 Heng Mui Keng Terrace, Singapore 119613 \\ wieslawdi2r.a-star.edu.sg
}

\begin{abstract}
This paper describes a rapid and automated method that extracts the fourth ventricle from MR brain images in normal and pathological subjects. Anatomical knowledge of fourth ventricle has been incorporated into the method to define a region of interest (ROI), determine intensity thresholds in the histogram of ROI, locate the starting point for the 3D region growing, and extract all parts of the fourth ventricle and aqueduct (even if they are disconnected on images as well as to avoid inclusion of non-ventricular cerebrospinal fluid regions). The method was validated qualitatively on $30 \mathrm{MRI}$ studies with variable parameters. The best overlap metric between a radiology expert and the method was 0.9988 and the worst 0.9621 on 10 quantitative studies. The mean and standard deviation of overlap metric were 0.9806 and 0.0105
\end{abstract}

\section{Introduction}

The normal fourth ventricle (V4) is remarkably symmetrical. It varies somewhat in size but little in shape. Since up to $20 \%$ brain tumors appear around the fourth ventricle region, subtle changes in displacement and deformity are important and helpful in detecting posterior fossa lesions [1]. The diagnosis of mass lesions, cerebellar atrophy, and inflammatory diseases depends on accurate measurement of the fourth ventricle size, shape and volume. Accurate evaluation requires identification of all portions of the fourth ventricle. Thus an objective, reproducible, and accurate extraction method of the fourth ventricle is necessary for its precise quantitative measurement. Several groups have reported methods for extraction of lateral ventricles and/or third ventricle based on various principles (Schnack et al. based on region-growing assisted by morphological operations 2001, [2]; Worth et al. applied thresholding, 1998 [3]; Baillard et al. used level sets, 2000 [4]; Wang et al. followed active models, 1998 [5]; Fisher, et al. imposed knowledge based methods, 2002 [6]). Only a few methods can extract the complete ventricular system including the fourth ventricle (Geraud, based on fuzzy set and information fusion, 1998 [7]; Hahn et al, applied region-growing, 1998 [8]) and there is no simple method for extracting the fourth ventricle suitable for clinical use. This may be due to several factors as user intervention may be required, there may be unsolved problems in attaining a fully automated fourth ventricle and aqueduct extraction, automated 
methods may not be thoroughly validated and disseminated, and the existing methods may be too slow for the accuracy of analysis needed to be clinically acceptable.

We have proposed a rapid and automated method to extract the complete human cerebral ventricular system from MR images [11]. This paper is an extension of our previous work towards more accurate and robust extraction of the fourth ventricle and quantitative validation of the approach.

\section{Material and Method}

V4 is surrounded by gray and white matter, and filled with cerebrospinal fluid (CSF). Theoretically, V4 could be extracted with one region-growing operation. Due to the partial volume effect, noise, and spatial and contrast resolutions of the scan, parts of the ventricle and aqueduct that connects V4 with third ventricle superiorly may not appear clearly on the images. Therefore, additional anatomical knowledge of the shape, position, and variability needs to be inserted into the method to define a ROI, determine intensity thresholds in the histogram of ROI, locate the starting point for the $3 \mathrm{D}$ region growing, and extract all parts of $\mathrm{V} 4$ and aqueduct, even if they are disconnected on images as well as to avoid inclusion of non-ventricular CSF regions.

\subsection{Define Region of Interest (ROI)}

The location of the Midsagital Plane (MSP) is extracted from the volume data [9], and the coordinates of the anterior commissure (AC) and posterior commissure (PC) are identified [10]. The raw data volume is repositioned into a coordinate system in a 3-D space (the coronal plane is $x z$, the sagittal plane is $y z$, the axial plane is $x y$ that parallels to the AC-PC line.

Taking into account the worst-case assumptions (the largest ROI necessary) based on literature studies of the fourth ventricle variability, a large rectangular ROI with width of $45 \mathrm{~mm}$ and height of $55 \mathrm{~mm}$ is defined on the MSP initially, Fig. 1 [1]. The ROI shape is changed to quadrangular in order to exclude the clivus, ambient cistern, quadrigeminal cistern, and cisterna magna.

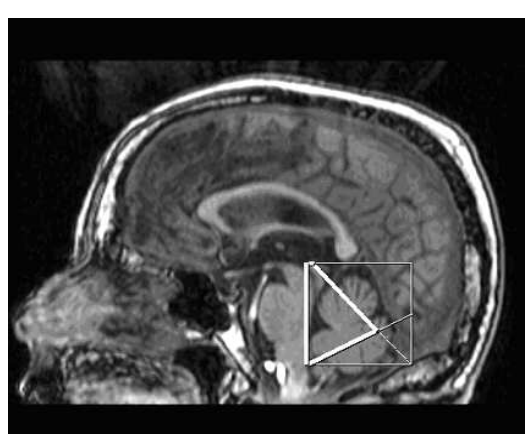

Fig. 1. ROI for the fourth ventricle

\subsection{Determine the Thresholds}

The thresholds for gray matter (GM), white matter (WM), and CSF are determined automatically by finding the peaks in the histogram of the ROI as follows:

- Calculate the histogram of ROI. This produces a multi-modal histogram where the intensity peaks and ranges can be chosen, corresponding to WM, GM, CSF, and other classes. 
- Smooth the histogram. The Fourier filter is applied to the original histogram to reduce the local variations. The smoothed histogram is with main peaks only and the noise is greatly reduced.

Identify the peaks in the histogram. The histogram is modeled as the sum of five smoothed, modified normal distribution (Gaussian) functions - one distribution each for CSF, CSF/GM, GM, GM/WM and WM.

- Fig. 2 shows a smoothed histogram distribution and the result of modeling each distribution separately by a Gaussian function. After modeling, the peaks, ranges and intersections of modeled distributions corresponding to CSF, GM, and WM can be taken based on neuroanatomical and radiological knowledge.

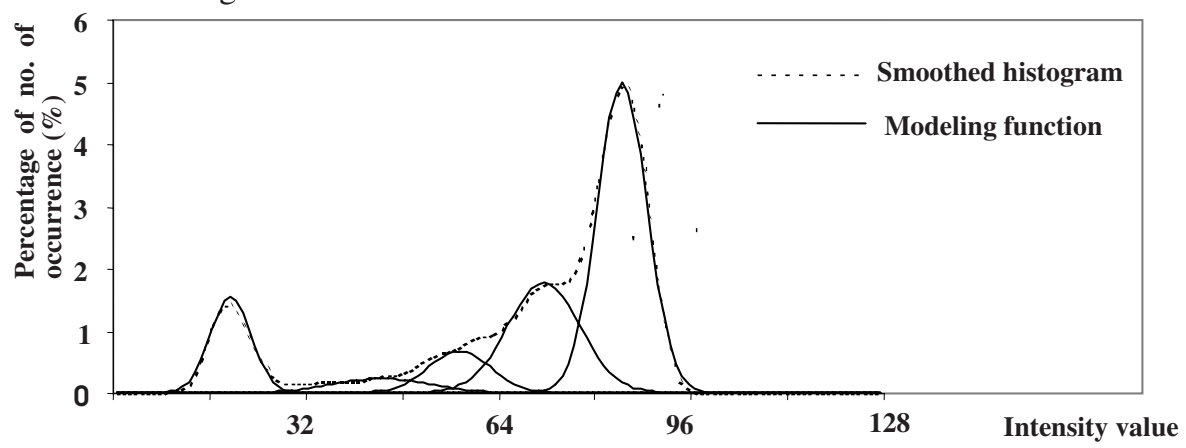

Fig. 2. The result of Gaussian fitting. The percentage of occurrence of grey value above 128 is less than $0.1 \%$ and is not shown.

The distribution of CSF, GM, and WM in some cases may be so small that they are not easily identifiable in the histogram of the initial ROI, which may hinder an accurate processing of the histogram. To improve the density distribution balance, the ROI size is reduced adaptively. And the histogram is recalculated and the new thresholds are obtained. The adaptive reduction of ROI is performed iteratively until the required distribution is achieved. Furthermore, the initial thresholds are changed adaptively during the growing of the fourth ventricle to cope with the partial volume effect and intensity inhomogeneity.

\subsection{Extract Fourth Ventricle}

V4 is subdivided into two subregions by the axial plane passing through the seed point located at the middle of the longest CSF segment on MSP, Fig. 3. This approach has several advantages:

- Region growing is simplified as simpler shapes replace complex ones.

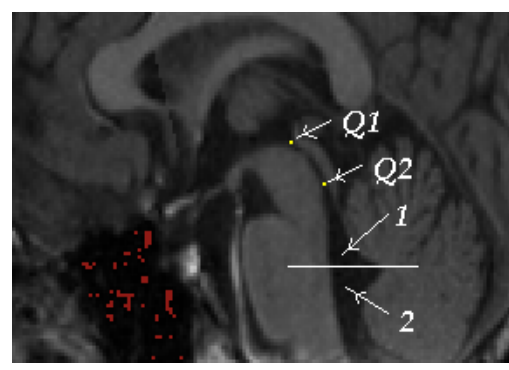

Fig. 3. Sub-regions of V4 
- Easier direction control regarding growing and connecting.

- Better leakage control and reduction of the partial volume effect, as it is easier to incorporate specific domain knowledge in each subregion.

- Processing is more efficient as only a subregion needs to be regrown in case of leakage.

- Easier to adjust the initial thresholds tailored to the local anatomy.

V4 is extracted on axial slices, superiorly in subregion 1 and inferiorly in subregion 2 starting from the axial slice containing the seed point, Fig. 3. The aqueduct (located between $Q_{1}$ and $Q_{2}$ ) cannot usually be extracted directly as its diameter is small (about $1.2 \mathrm{~mm}$ on average). Then, the aqueduct is taken as the voxels with the lowest intensity in the axial region confined by $Q_{1}$ and $Q_{2}$ on T1-weighted images (or the highest on T2-weighted images) when extracting the subregion 1 of V4.

The following leakages may occur from V4: 1) dorsoposteriorly through the superior medullary velum to the cisterna ambiens, 2) ventroposteriorly through the inferior medullary velum to the cisterna magna. To prevent leakage to the cisterna ambiens, two initial constraints are imposed:

- The length of CSF segment on the MSP of the current axial slice should not be greater than that in the previous slice in superior direction.

- The distance between the mass center of the grown region and the MSP should be less than $2 \mathrm{~mm}$ at sagittal direction. The mass center of the foreground region should not deviate more than $2 \mathrm{~mm}$ away from that of the previous slice at sagittal direction (Newton et al., 1978).

To prevent the leakage to the cisterna magna, the width of the foreground region at the lateral recesses should be less than $2 \mathrm{~mm}$ at sagittal direction [1].

When a sudden appearance of a large connected area appears in the abovementioned areas, it is possibly a leak into a cistern. When the leakage occurs, the voxels of this leakage region are set to a value that is outside of CSF range (to prevent them from leaking in any subsequent region growing). Also, the CSF intensity range (threshold) is narrowed and the last region-growing step is repeated and checked whether the leakage is controlled. This procedure is repeated until all correct growing is completed.

\section{Validation}

The V4 extraction method is implemented on the Windows platform using C++ program. First evaluation of the method was done on a T1-weighted MR brain study, with volume size of 256x256x168, voxel size of 1.0x1.0x0.67 mm, of good image quality and known underlying extraction result of fourth ventricle. The run-time of the extraction method was less than 5 seconds on a Pentium $3800 \mathrm{MHz}$ PC with $128 \mathrm{MB}$ of RAM. Fig. 4 shows the extraction results on some axial slices.

To test the validity and applicability of the method, 30 real clinical MR volume data were collected from different sources. The volume data were acquired using a T1-weighted spin echo and a Spoiled Gradient Echo Recovery (SPGR) sequence. They included healthy controls and 6 patients with various pathologies, age ranging from 12 to 60 years, both females and males. The volume size of the scans ranged 
from $192 \times 256 \times 192$ to $256 \times 181 \times 256$, the voxel size from 0.897 to $1 \mathrm{~mm}$ in sagittal, from 0.879 to $2.0 \mathrm{~mm}$ in coronal, and 0.67 to $3.5 \mathrm{~mm}$ in axial directions. Some of the volume data had significant partial volume effect and inhomogeneity. All of images were not corrected for scanner RF field nouniformity.

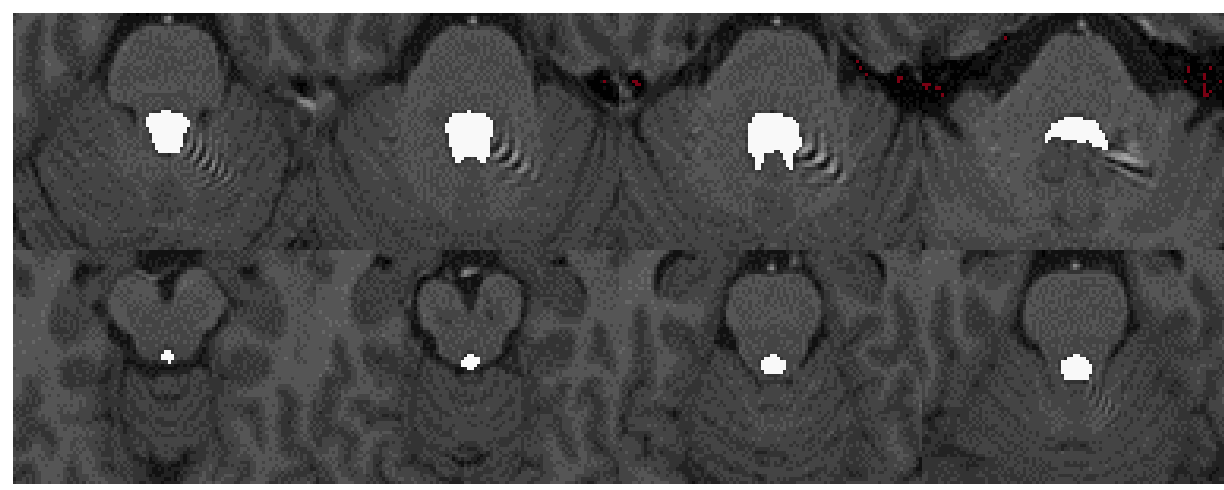

Fig. 4. Eight axial slices showing the fourth ventricle extracted

The run time of the extraction method was less than 5 seconds for all of the data on a Pentium3 $800 \mathrm{MHz}$ with $128 \mathrm{MB}$ RAM PC regardless of the complexity of V4 and the severity of the leakages. The method for automatic determination of intensity model parameters using statistics worked well even for a wide variety of scan data sets obtained at different times from different scanners under different protocols. In all tested cases the method succeeded in finding and extracting V4. These tests showed the method yielded qualitatively good

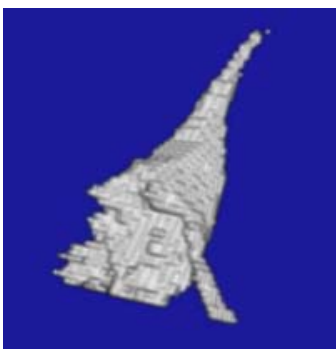

Voxel size: $1.0 \times 1.0 \times 0.67$

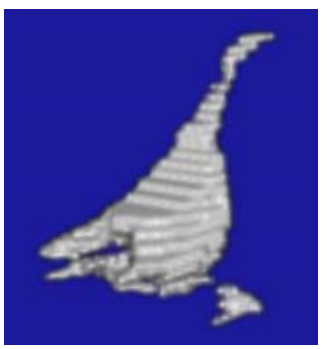

Voxel size:0.898x0.898x1.238

Fig. 5. Three-dimensional rendering of extracted V4 results (visual check of the extraction) for the fourth ventricle, Fig. 4 and Fig. 5.

In order to quantitatively evaluate the method, an experienced radiology expert randomly selected 10 volume data from the tested cases and extracted the fourth ventricle manually. To quantitatively compare both radiology expert and the method extraction results, the overlap metric was calculated:

$$
\varepsilon=2 * V_{12} /\left(V_{1}+V_{2}\right)
$$

where $V_{1}$ and $V_{2}$ are the volumes of the two extractions that are compared, and $V_{12}$ is the volume of the overlap of the two extractions. The false negative (missed) and false positive (over-estimated) volumes of the method extraction were calculated; the ratios 
of the false negative/the false positive to overlap were calculated as well. Some results are shown in Table 1 , the unit is 1 voxel.

We calculated overlap metric $\varepsilon$ between automated method and expert extractions. For 10 cases, the best overlap metric $\varepsilon$ was 0.9988 and the worst was 0.96211 . The mean and standard deviation of overlap metric on 10 cases were 0.9806 and 0.0105 .

Table 1. Comparision of radiology expert and the method extraction results (unit is 1 voxel)

\begin{tabular}{|l|l|l|l|l|l|}
\hline VoxelSize & ExpertResult & ExtractResult & Overlap & FalseNegative & FalsePositive \\
\hline $1.0 \times 1.0 \times 0.67$ & 3603 & 3658 & $3571(0.9836)$ & $32(0.00896)$ & $87(0.02436)$ \\
\hline $1.0 * 1.0 * 1.0$ & 868 & 891 & $866(0.98465)$ & $2(0.00231)$ & $25(0.02887)$ \\
\hline $0.9375 \times 0.9375 \times 3.0$ & 711 & 746 & $710(0.97461)$ & $1(0.00141)$ & $36(0.0507)$ \\
\hline $0.879 * 0.879 * 1.953$ & 1255 & 1252 & $1252(0.9988)$ & $3(0.00240)$ & $0(0.0)$ \\
\hline $0.898 * 0.898 * 1.33$ & 1077 & 1079 & $1060(0.9833)$ & $17(0.01604)$ & $19(0.01792)$ \\
\hline $0.879 * 0.879 * 0.9$ & 1779 & 1863 & $1752(0.9621)$ & $27(0.01541)$ & $111(0.06336)$ \\
\hline $0.879 * 0.879 * 0.9$ & 3137 & 3234 & $3130(0.9826)$ & $7(0.00224)$ & $104(0.03323)$ \\
\hline $0.898 \times 0.898 \times 1.238$ & 1304 & 1267 & $1257(0.9779)$ & $47(0.03739)$ & $10(0.00796)$ \\
\hline $0.9375 * 0.9375 * 1.5$ & 1054 & 1086 & $1036(0.9682)$ & $18(0.01737)$ & $50(0.04826)$ \\
\hline $0.879 * 0.879 * 1.953$ & 1145 & 1134 & $1128(0.9899)$ & $17(0.01507)$ & $6(0.00532)$ \\
\hline
\end{tabular}

We analyzed why a large part of the voxels were over-estimated by the method for the worst case. One reason is ventricle boundary that is not clear due to partial volume effect; another reason is the expert did the job in 2-D space and did not extract very small parts of V4 in many cases, Fig. 6. Our method did the extraction in 3-D space and had the capability in extracting the subtle parts of V4. So, the false positive voxels caused by later reason should be correct result (black arrows, Fig. 6).
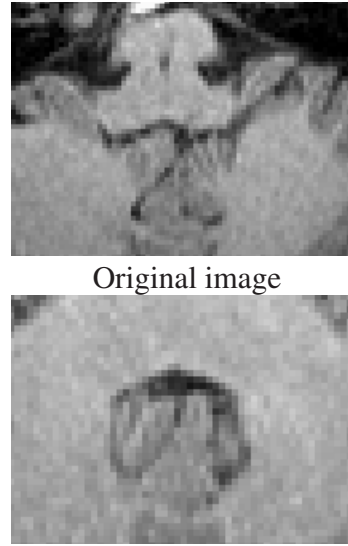

Original image

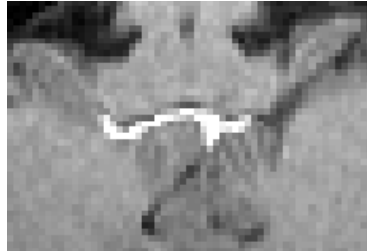

Expert extraction

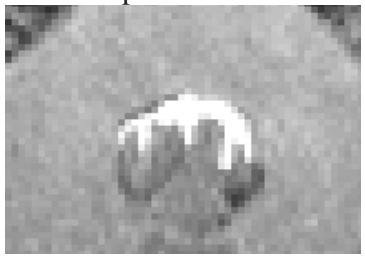

Expert extraction

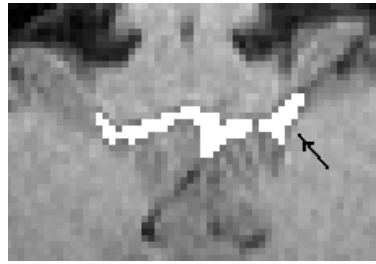

Method extraction

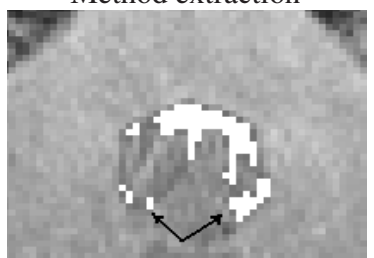

Method extraction

Fig. 6. The reason for high "false positive"

The testing shows the method has the capability to extract V4 and aqueduct on images that are much different from the images that were used to develop the method initially. The brains of children are smaller and of different shape than adult brains, and the brains of the patients with various pathologies deviate even more in size, shape, and relative constitution of brain and CSF. The method can handle these cases and extract V4 with good qualitative and quantitative results. 


\section{Discussion}

Anatomical knowledge of the fourth ventricle has been incorporated into the method to find all constituting parts of the ventricle and aqueduct, even if they are not visible clearly in 2D images, and to avoid inclusion of nonventriclular CSF regions. The validation was performed on a data set of 30 T1-weight and SPGR MR brain images. The volume data included healthy controls and 6 patients with various pathologies, age ranging from 12 to 60 years, both females and males. The method yielded useful results for all of the images; the best overlap metric $\varepsilon$ between a radiology expert and the method was 0.9988 and the worst was 0.96211 for 10 studies; the mean and standard deviation of overlap metric were 0.9806 and 0.0105 . These tests showed that the method gives qualitatively and quantitatively valid fourth ventricle extraction, and a few non-valid results were easily detected and corrected. Also, the tests showed that method had the capability in extracting the subtle parts of V4 and yielded the extraction result better than the expert at some parts.

Our method is based on region-growing and anatomical knowledge. When the slice thickness, especially in coronal and axial directions is too big, the method cannot get a good extraction result. The tests showed that the method would run correctly when the slice thickness is less than $3.5 \mathrm{~mm}$ at axial direction or is less than $2.0 \mathrm{~mm}$ at coronal direction. A bigger slice thickness would increase the partial volume averaging which has a significant effect on the algorithm accuracy. The method assumes that the MSP has been generated and the AC and PC landmarks have been detected. The errors calculating MSP and detecting the $\mathrm{AC}$ and $\mathrm{PC}$ are propagated in the method, and will influence on the extraction accuracy. The AC detection error will be of a serious influence on the extraction result. Also, noise had a more notable effect than partial volume and RF inhomogeneity on the method because the method is driven by anatomic knowledge and statistics.

Our emphasis here is to automate an established, precise extraction procedure for V4. The utility of the method to the general image processing is to deal directly with problems by using extensive domain knowledge. This method shows an example of the application of anatomic knowledge (expectations about tissue response in MR such as knowing which peak in the histogram to use in which situation; specific sequence of successive region extraction and detection of leakages and connection, Fig. 7) along with image processing and pattern recognition methods (such as locating peaks using the Gaussian fitting and directional region growing from a starting point).

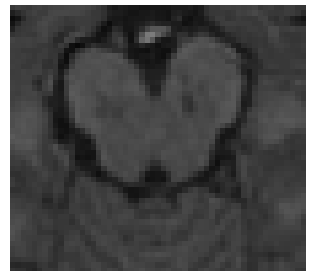

Original image

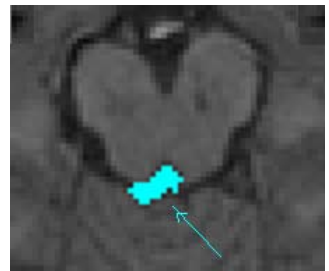

Leakage

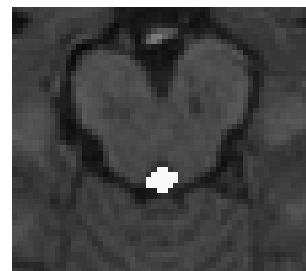

Leakage-control

Fig. 7. Leakage control 
We have examined the method for the extraction of the fourth ventricle qualitatively and quantitatively. The tested result shows that the method makes a good base for further building the relationship between some brain diseases and the patterns of fourth ventricle, determining the relationship between age and absolute volumes of the fourth ventricle. The assessment of the absolute volume and 3D-morphology assessment of the fourth ventricle may represent a more objective way to determine normal and abnormal development to clinical subjects. The fourth ventricle is a small structure with volume as little as $0.03 \mathrm{cc}$, hence the evaluation is more demanding. This relationship between the volume of the fourth ventricle and its estimation using our protocol can be further validated with a larger number of subjects, and will facilitate the detection of ventricular pathology.

\section{Conclusion}

We have presented a rapid and automated method to extract human fourth ventricle on MRI driven by data and anatomic knowledge. Use of anatomical knowledge in the formulation of the method is advantageous as it aids to identify the thresholds of extraction, localize fourth ventricle structure and to guide the ventricle extraction more reliably. The proposed method yielded useful results for all of the tested images; the best overlap metric $\varepsilon$ between a radiology expert and the method was 0.9988 and the worst 0.96211 on 10 quantitative studies. The mean and standard deviation of overlap metric were 0.9806 and 0.0105 . These tests showed that the method gave a good qualitative and quantitative result for the extraction of fourth ventricle even in the presence of significant inhomogeneity and partial volume effect.

\section{References}

1. Newton TH and Potts DG (eds) Radiology of the skull and brain, ventricles and cisterns. MediBooks, Great Neck, NY, pp. 3303-3397.

2. Schnack HG, Hulshoff PHE, Baare WFC, Viergever MA, Kahn RS, "Automatic extractation of the ventricular system from MR images of the human brain," NeuroImage 2001 vol. 14, pp. 95-104.

3. Worth AJ, Makris N, Patti MR, Goodman JM, Hoge EA, Caviness VS, Kennedy DN, "Precise extractation of the lateral ventricles and caudate nucleus in MR brain images using anatomically driven histograms," IEEE Transactions on Medical Imaging 1998, vol. 17, no. 2, pp. 303-310.

4. Baillard C, Hellier P, Barillot C, "Extractation of 3D brain structures using level sets and dense registration," IEEE Workshop on Mathematical Methods on Biomedical Image Analysis (MMBIA 2000), pp. 94-101.

5. Wang Y, Staib LH, "Boundary finding with correspondence using statistical shape models," Proceeding IEEE conference of computer vision and pattern recognition 1998, pp. 338-345.

6. Fisher E, Rudick RA, "Method and system for brain volume analysis," USA patent US006366797B1, 2002.

7. Geraud T, 1998. http://www-sig.enst.fr/tsi/groups/TII/active 
8. http://www.mevis.de/projects/volumetry/volumetry.html, Center for Medical Diagnostic Systems and Visualisation, University of Bremen.

9. $\mathrm{Hu} \mathrm{QM}$, Nowinski WL, "A rapid algorithm for robust and automatic extraction of the midsagittal plane of the human cerebrum from neuroimages based on local symmetry and outlier removal", NeuroImage (under revision).

10. Nowinski WL, "Modified Talairach landmarks", Acta Neurochirurgica, 2001; 143(10): $1045-1057$.

11. Xia Y, Hu QM, Aziz Aamer, Nowinski WL, "Knowledge-Driven Automated Extraction of the Human Cerebral Ventricular System from MR Images", In: Taylor C and Noble A (eds), Proc. Information Processing in Medical Imaging IPMI'03,LNCS, 2003; 271-281. 